\title{
Environmental sampling of the severe acute respiratory syndrome coronavirus 2 Delta variant in the inpatient wards of a hospital in Nanjing
}

\author{
Ming Chu ${ }^{1 \#}$, Minjuan $\mathrm{Chu}^{2 \#}$, Yiping Mao ${ }^{3}$, Zhanjie $\mathrm{Li}^{1}$, Shuangyuan Liu ${ }^{1}$, Hui Liu ${ }^{1}$, Jingwu Ge ${ }^{1}$, Zhihang Peng ${ }^{4}$, \\ Yongfeng Yang ${ }^{5}$, Yun Liu ${ }^{1}$, Jinhai Tang ${ }^{1}$
}

${ }^{1}$ Department of Infection Management, The First Affiliated Hospital of Nanjing Medical University, Nanjing, China; ${ }^{2}$ Department of Critical Care Medicine, The First Affiliated Hospital of Nanjing Medical University, Nanjing, China; ${ }^{3}$ Department of Infection Management, The Affiliated Hospital of Xuzhou Medical University, Xuzhou, China; ${ }^{4}$ Department of Epidemiology and Biostatistics, School of Public Health, Nanjing Medical University, Nanjing, China; ${ }^{5}$ Center of Infectious Diseases, Affiliated Nanjing Hospital of Nanjing University of Chinese Medicine (The No. 2 Hospital of Nanjing), Nanjing, China

Contributions: (I) Conception and design: M Chu, Y Mao, J Tang; (II) Administrative support: M Chu, Y Liu; (III) Provision of study materials: Z Li, S Liu, H Liu, Y Yang, M Chu; (IV) Collection and assembly of data: J Ge, Z Peng, M Chu; (V) Manuscript writing: All authors; (VI) Final approval of manuscript: All authors.

\#These authors contributed equally to this work.

Correspondence to: Jinhai Tang, MD; Yun Liu, MD. Department of Infection Management, The First Affiliated Hospital of Nanjing Medical University, Nanjing 210009, China. Email: jhtang@njmu.edu.cn; liuyun@njmu.edu.cn.

Background: Little is known about severe acute respiratory syndrome coronavirus 2 (SARS-CoV-2) Delta variant of concern (VOC)-contaminated environmental surfaces and air in hospital wards admitting COVID-19 cases. Our study was designed to identify high-risk areas of Delta VOC contamination in the hospital and provide suggestions to in-hospital infection control. We analyzed the SARS-CoV-2 Delta VOC contamination in the air and environmental surface samples collected from a hospital in Nanjing, China.

Methods: We collected data on clinical features, laboratory tests, swab tests, and hospital wards, identified the factors associated with environmental contamination, and analyzed patients' hygiene behaviors during hospitalization.

Results: A total of 283 environmental surface and air samples were collected from a hospital admitting 36 COVID-19 patients. Twelve swab samples from ten patients were positive. Toilet seats had the highest contamination rate $(11.8 \%)$, followed by bedside tables $(8.2 \%)$, garbage bins $(5.9 \%)$, and bedrails $(1.6 \%)$. The median time of symptom onset to surface sampling was shorter in the positive environment group than in the negative environment group (11 vs. 18 days; $\mathrm{P}=0.001)$. The results indicated that environmental surface contamination was associated with positive anal swabs [odds ratio (OR) 27.183; 95\% CI: 2.359226.063; $\mathrm{P}=0.003$ ] and the time from symptom onset to surface sampling (OR 0.801; 95\% CI: 0.501-0.990; $\mathrm{P}=0.046$ ). The survey revealed that $33.3 \%$ of the patients never cleaned or disinfected their bedside tables or toilets, and $8.3 \%$ of them only cleaned their bedside tables or toilets. More than half of the patients often $(25 \%)$ or always $(30.6 \%)$ put the used masks on their bedside tables. Only $16.7 \%$ of the patients threw the masks into the specific garbage bin for used masks.

Conclusions: The SARS-CoV-2 Delta VOC was detected on environmental surfaces, especially toilet seats and bedside tables, within a median time of 11 days after symptom onset. Our study provided potential predictors for environmental surface contamination, including positive anal swabs and the time from symptom onset to sampling. Disinfecting high-risk environmental surfaces should be emphasized in hospital wards, especially for patients in the early stage of COVID-19.

Keywords: 2019 coronavirus disease (COVID-19); Delta variant; environmental 
Submitted Sep 27, 2021. Accepted for publication Oct 31, 2021.

doi: $10.21037 / \mathrm{atm}-21-5147$

View this article at: https://dx.doi.org/10.21037/atm-21-5147

\section{Introduction}

Since its outbreak in 2019, the 2019 coronavirus disease (COVID-19) has spread across the world. As of September 6, 2021, there have been 220.56 million confirmed cases of COVID-19, including 4.57 million deaths globally (1). Since July 20, 2021, more than 820 individuals have been infected by the severe acute respiratory syndrome coronavirus 2 (SARS-CoV-2) Delta variant. The SARSCoV-2 Delta variant (B.1.617.2), first identified in India in October 2020, was classified as a variant of concern (VOC) on May 11, 2021, and was subsequently labeled the Delta variant by the World Health Organization under a new naming system introduced on May 31, 2021 (2). From midApril 2021 onward, the Delta variant has spread rapidly and became the dominant variant in England by late May (3).

The Delta variant is more virulent than are the previous variants (4). The COVID-19 cases infected with the Delta variant face a 2-times greater risk of hospitalization compared with those infected by the Alpha variant (5). The widespread Delta variant (6) with an increased viral load (7) can infect both vaccinated and unvaccinated populations (8), indicating a potential risk of illness even after vaccination (9). Therefore, it is necessary to evaluate the level of Delta VOC in an environment in which infected patients live.

Although COVID-19 is transmitted mainly through respiratory droplets and close contact (10), infection may occur by touching SARS-CoV-2-contaminated objects (11). Hence, environmental contamination has also aroused concerns around the world. COVID-19 patients can contaminate various areas in wards, including the bedrails, pillows, bed sheets, and air exhaust outlets (12). SARS-CoV-2 can be detected in the toilet (13), positive environmental samples from which have been associated with positive anal swabs (14). The analysis of environmental samples has provided evidence that SARS-CoV-2 can be transmitted through the surfaces of mobile phones or other patient belongings $(14,15)$. However, no previous studies have evaluated the environmental contamination in hospital wards of Delta variant-infected patients.

In this study, we detected the Delta variant in the environmental samples collected from hospital wards. Our objectives were to (I) explore the characteristics of the environmental contamination in hospital wards for Delta VOC-infected cases, (II) assess the factors associated with positive environmental samples, and (III) investigate the patients' hygiene behaviors during hospitalization. We present the following article in accordance with the Materials Design Analysis Reporting (MDAR) checklist (available at https://dx.doi.org/10.21037/atm-21-5147).

\section{Methods}

\section{Study design and setting}

This study was conducted in the nonnegative pressure general wards of Nanjing No. 2 Hospital. The wards were put in use on August 14, 2021. On 20 August, 2021, a total of 25 COVID-19 cases were hospitalized into 15 wards, including 10 two-bed rooms and 5 single-bed rooms, and the first environmental and biological sampling was performed. On August 21, we sampled the bedside air and ward toilets of cases with positive environmental samples. On August 27, a total of 36 COVID-19 cases were hospitalized into 19 wards, including 17 two-bed rooms and 2 single-bed rooms. We performed the second environmental sampling (Figure 1) and collected the biological samples from the patients. On the same day, we conducted a questionnaire survey on the 36 patients to investigate their hygiene behaviors during hospitalization after obtaining informed consent.

\section{Sample collection}

Biological samples, including throat and anal swabs, were collected from the hospitalized patients for daily COVID-19 nucleic acid testing in the morning. The nurses cleaned and disinfected the ward passages and the floor of the nurse station with $1,000 \mathrm{mg} /$ liter of chlorine solution and wiped the surfaces with chlorine-containing disinfectant wipes twice daily. The nurses disinfected the lids, inside and outside of the garbage bins, and contents inside the bins with $2,000 \mathrm{mg} /$ liter of chlorine solution before collecting the garbage. The surfaces and floors of the wards, including ward toilets, were disinfected. Each day, before the first cleaning, we sampled the frequently touched surfaces 


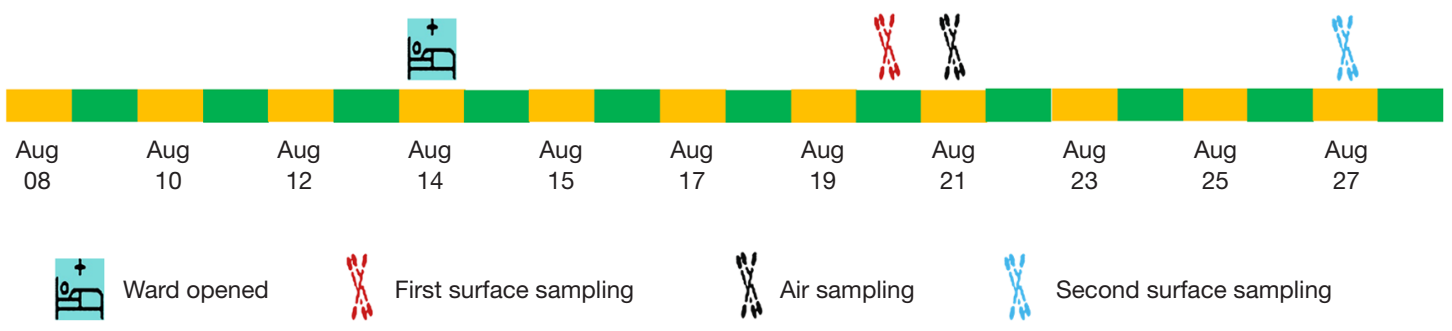

Figure 1 The flowchart of this study.

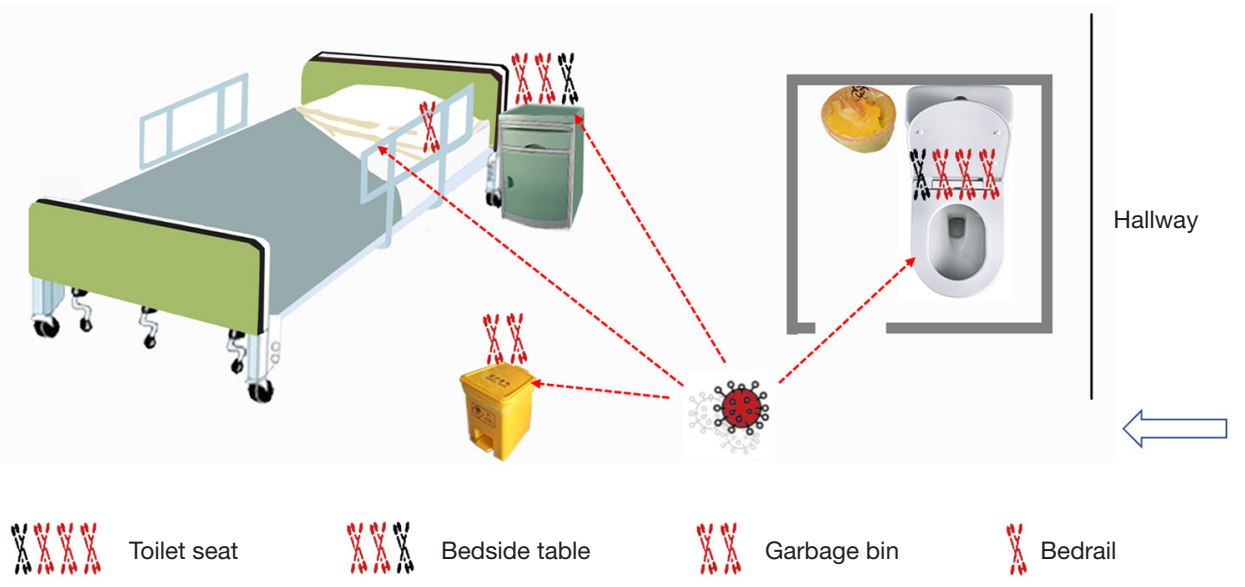

Figure 2 The environmental sampling in the hospital ward.

in the wards and the public areas. Samples in the wards were collected from the bedside tables, bedrails, patients' mobile phones, garbage bins, and toilet seats (Figure 2). Samples in the public areas were collected from the garbage bins in the hallway, mobile treatment carts, armrests and electrocardiographs, mobile phones of physicians and nurses, keyboards, telephones, and desktops in the nurse station. For larger surfaces, such as bedside tables, the samples were collected from a minimum area of $100 \mathrm{~cm}^{2}$, and for smaller surfaces, such as mobile phones, the samples were collected in from an as-large-as-possible area. Samples were collected using sterile swabs (Yocon, Beijing, China) and kept in virus preservation solution (Yocon, Beijing, China).

The air in the room and toilet in each ward was disinfected with UV lamps by nurses twice a day, 1 hour each time. We sampled the air before disinfection using Aerosol Particle Liquid Concentrator (model WA-400II, Beijing Dingblue Technology Co., Ltd., Beijing, China) at $400 \mathrm{~L} / \mathrm{min}$ for 20 minutes (16). Each air sample was collected and stored in $3 \mathrm{~mL}$ of the virus sampling liquid mentioned above.

\section{Questionnaire survey}

The questionnaire was constructed using a mobile app named "Wenjuanxing" (www.wjx.cn) and used in face-to-face interviews in the wards after patients' informed consent was obtained. The questions covered cleaning and disinfection of bedside tables and toilets, mask disposal behaviors, and disinfection of mobile phones during hospitalization. The 4-point Likert scale (never, occasionally, often, every time) was used for all questions (17).

\section{Viral RNA detection by reverse transcription-polymerase chain reaction}

Following standard procedures, the environmental samples were collected by well-trained medical staff and stored in virus medium. Viral RNA was extracted within 2 hours using the Nucleic Acid Isolation Kit (Jiangsu Bioperfectus Technologies Co., Ltd., Taizhou, China) according to the 
manufacturer's instructions. Reverse transcription-polymerase chain reaction (RT-PCR) was conducted with primers and probes targeting $N$ and $O R F 1 a / b$ genes, and a positive reference gene by using the RNA Detection Kit for SARSCoV-2 (Jiangsu Bioperfectus Technologies Co., Ltd.) (18). The reaction cycles followed the manufacturer's specifications (Bioperfectus Technologies Co., Ltd.). The detection limit of cycle threshold $(\mathrm{Ct})$ was set at $40(500$ copies $/ \mathrm{mL})$. The samples were only considered to be positive when the Ct values of both the $N$ and ORF1a/b genes were $\leq 40$. However, a specimen that tested positive for only 1 gene was reported as "weakly positive". All tests were performed under strict biosafety conditions following standard operating procedures.

\section{Statistical analyses}

All participants were divided into the group of positive environmental surface sampling results and the group of negative environmental surface sampling results. Categorical variables are expressed as numbers and percentages and were compared using Pearson $\chi^{2}$ tests. Continuous variables are presented as mean \pm standard deviation (SD) or medians and interquartile ranges (IQRs) and were compared using $t$-tests or Wilcoxon rank-sum tests if the data were not normally distributed. Generalized linear mixed models (GLMMs) adjusted for age, sex, sampling site (throat or anus), and time from symptom onset to surface sampling were employed to investigate the effect of swab positivity on environmental surface sampling results in multivariate analysis. A multivariable generalized linear mixed model was established to identify the associations between risk factors and positive environmental sampling results. The odds ratios (ORs) and corresponding 95\% confidence intervals (CIs) were calculated to assess these associations. SPSS software version 26.0 (IBM Corporation, Armonk, NY, USA) was used for all statistical analyses. A P value $<0.05$ was considered statistically significant.

\section{Ethics statement}

The study was conducted in accordance with the Declaration of Helsinki (as revised in 2013). Our study protocol was approved by the Ethics Committee of The No. 2 Hospital of Nanjing (No. 2021-ls-ky030). Informed consent was obtained from the patients before the questionnaire survey was administered. No personal information was obtained in this study.

\section{Results}

\section{Characteristics of 36 COVID-19 cases}

Table 1 shows the characteristics of 36 COVID-19 cases. Their median age was 39.5 years (IQR, 26.3-58 years), and $21(58.3 \%)$ were male. All 36 cases were infected in Yangzhou and then admitted to Nanjing No. 2 Hospital, Jiangsu Province. We discovered that 15 (15/36, 41.7\%) cases had received 2 doses of vaccination, $9(9 / 36,25 \%) 1$ dose of vaccination, and $12(12 / 36,33.3 \%)$ no vaccination. Of the 36 cases, 2 cases $(2 / 36,5.6 \%)$ were classified as mild and $34(34 / 36,94.4 \%)$ as moderate. All $(36 / 36,100.0 \%)$ were cured and discharged. The common symptoms at onset were fever $(12 / 36,33.3 \%)$, cough or dry cough (23/36, 63.9\%), throat discomfort $(8 / 36,22.2 \%)$, fatigue (6/36, 16.7\%), and hyposmia (3/36, 8.3\%). Pulmonary computerized tomography (CT) abnormalities were found in $34(34 / 36,94.4 \%)$ cases.

The median time from symptom onset to the first surface sampling was 11 days. In the first sampling, among the throat and anal swabs collected from 25 patients, 25 throat samples were positive and 10 anal samples were positive. The median time from symptom onset to the second surface sampling was 19.5 days. In the second sampling, among the throat and anal swabs collected from 36 patients, 13 throat samples were positive and 14 throat samples were weakly positive, while 3 anal samples were positive and 3 anal samples were weakly positive.

\section{Environmental samples of 39 COVID-19 cases}

Among the 283 surface and air samples collected from 36 COVID-19 cases, 12 (12/283, 4.2\%) samples from 10 COVID-19 cases (10/36, 27.8\%) tested positive according to RT-PCR. All 12 positive samples were collected from these 10 patients' wards, including $5(5 / 61,8.2 \%)$ from bedside tables, $4(4 / 34,11.8 \%)$ from toilet seats, 2 (2/34, $5.9 \%)$ from garbage bins, and $1(1 / 61,1.6 \%)$ from bedrails. By contrast, all the samples from patients' mobile phones $(0 / 61,0.0 \%)$ and public areas $(0 / 20,0.0 \%$; including passages and the nurse station) tested negative according to RT-PCR (Table 2). All the air samples (0/12, $0 \%$ ) from bedsides and toilets in 6 positive wards were negative.

\section{Positive environmental samples from 10 COVID-19 cases}

A total of 56 samples were collected from 10 COVID-19 cases who had at least 1 positive or weakly positive 
Table 1 Baseline characteristics of the study population

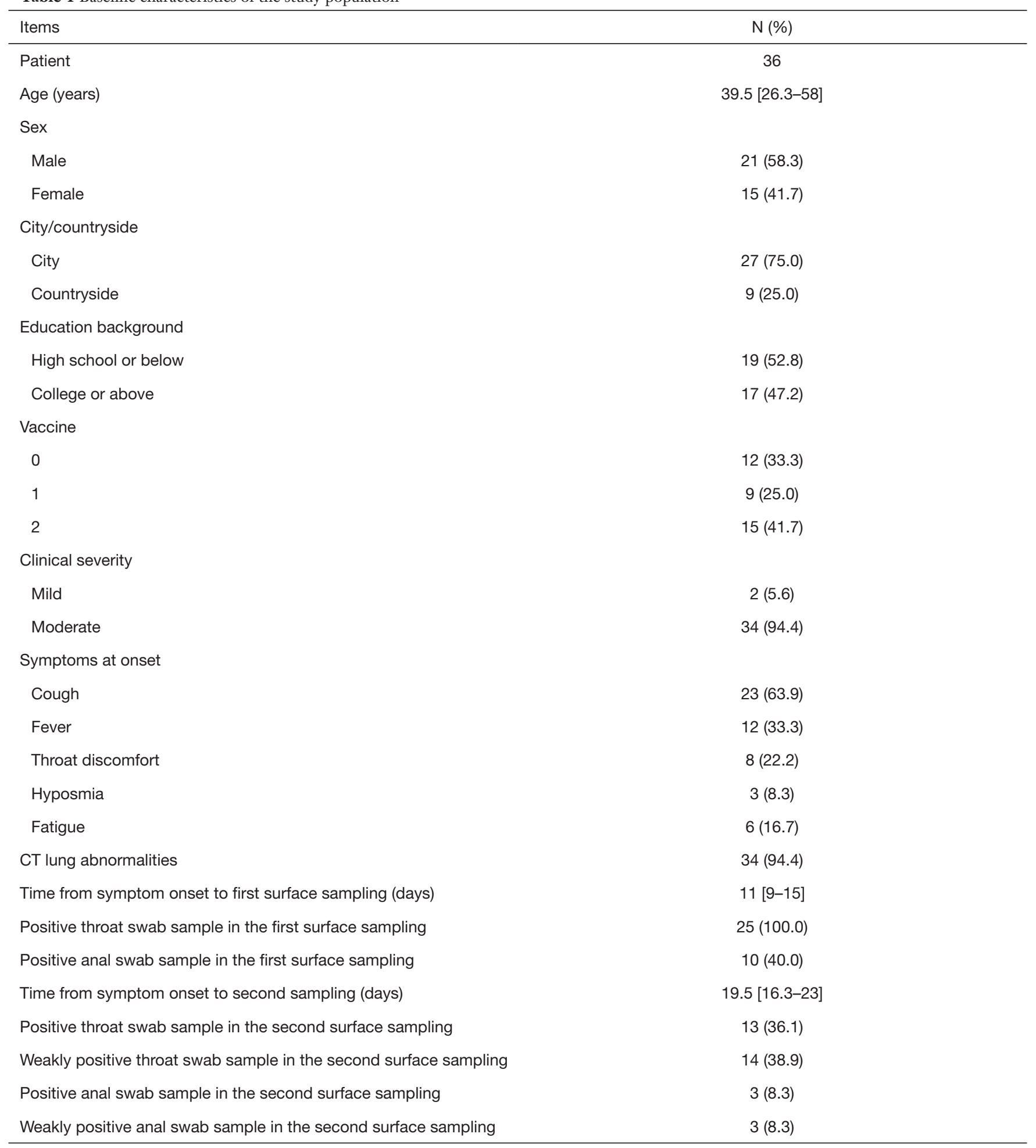

$\mathrm{CT}$, computed tomography. 
Table 2 Environmental specimens from 36 COVID-19 cases

\begin{tabular}{|c|c|c|c|}
\hline Variable & Sampling date & $\begin{array}{l}\text { No. of positive or weakly positive } \\
\text { environmental samples (\%) }\end{array}$ & $\begin{array}{l}\text { No. of environmental samples } \\
\text { collected }\end{array}$ \\
\hline \multicolumn{4}{|l|}{ First surface sampling } \\
\hline Bedside table & $08-20$ & $4(6.6)$ & 25 \\
\hline Bedrail & $08-20$ & $1(1.6)$ & 25 \\
\hline Toilet seat & $08-20$ & $4(11.8)$ & 15 \\
\hline Patient's mobile phone & $08-20$ & 0 & 25 \\
\hline \multicolumn{4}{|l|}{ Public area } \\
\hline Hallway & $08-20$ & 0 & 4 \\
\hline Toilet (air) & $08-21$ & 0 & 6 \\
\hline \multicolumn{4}{|l|}{ Second surface sampling } \\
\hline Bedside table & $08-27$ & $1(1.6)$ & 36 \\
\hline Bedrail & $08-27$ & 0 & 36 \\
\hline Garbage bin & $08-27$ & 0 & 19 \\
\hline Toilet seat & $08-27$ & 0 & 19 \\
\hline Patient's mobile phone & $08-27$ & 0 & 36 \\
\hline
\end{tabular}

environmental sample (Figure 3). Among them, 12 samples $(12 / 56,21.4 \%)$ tested positive or weakly positive according to RT-PCR (Figure 3). Among the 12 positive samples, 5 $(5 / 10,50 \%)$ were taken from bedside tables, $4(4 / 7,57.1 \%)$ from toilet seats, $2(2 / 7,28.6 \%)$ from garbage bins, and 1 $(1 / 10,10 \%)$ from bedrails. Interestingly, 2 samples from garbage bins were positive, while the other 10 were weakly positive. All throat swab samples from these 10 patients were positive. The positive rate of patients' anal swabs was $70 \%$, and the weakly positive rate was only $10 \%$.

\section{Association between risk factors of COVID-19 cases and positive environmental samples}

The patient's throat and anal swabs were sampled simultaneously on the day of environmental surface sampling. A total of 61 patient-related samples were obtained from 2 rounds of surface sampling, including 25 cases in the first round and 36 cases in the second round. The environmental surface samples were collected from patients' bedside tables, bed rails, mobile phones, garbage bins, and toilets. The positivity of any of these samples indicated the positivity of the environmental surface sampling.

Statistically significant differences were found between the positive and negative environments in throat swab samples $(\mathrm{P}=0.005)$, anal swab samples $(\mathrm{P}<0.001)$, and the time from symptom onset to surface sampling $(\mathrm{P}=0.001$; Table 3). The positive rate of throat swab samples was higher in the positive environmental sample group than in the negative environmental sample group $(100 \%$ s. $54.9 \% ; \mathrm{P}=0.005)$. The positive rate of anal swab sample 


\begin{tabular}{|c|c|c|c|c|c|c|c|c|c|c|c|c|c|c|c|c|c|}
\hline \multirow[b]{2}{*}{ ID } & \multirow[b]{2}{*}{ Room } & \multirow[b]{2}{*}{$\begin{array}{c}\text { Date of } \\
\text { symptom } \\
\text { onset }\end{array}$} & \multirow[b]{2}{*}{$\begin{array}{l}\text { Date of } \\
\text { surface } \\
\text { sampling }\end{array}$} & \multirow{2}{*}{$\begin{array}{c}\text { Time of } \\
\text { symptom } \\
\text { onset to } \\
\text { surface } \\
\text { sampling } \\
\end{array}$} & \multirow[b]{2}{*}{ Sex } & \multirow[b]{2}{*}{ Age } & \multirow[b]{2}{*}{ Severity } & \multirow[b]{2}{*}{ Vaccine } & \multirow{2}{*}{$\begin{array}{l}\text { Throat swab } \\
\text { on surface } \\
\text { sampling day } \\
\text { (Ct value) }\end{array}$} & \multirow{2}{*}{$\begin{array}{c}\text { Anal swab } \\
\text { on surface } \\
\text { sampling } \\
\text { day } \\
\text { (Ct value) }\end{array}$} & \multicolumn{5}{|c|}{ Surface sample in hospital ward } & \multicolumn{2}{|c|}{ Air sample } \\
\hline & & & & & & & & & & & $\begin{array}{c}\text { Bedside table } \\
\text { (Ct value) }\end{array}$ & $\begin{array}{c}\text { Bedrail } \\
\text { (Ct value) }\end{array}$ & $\begin{array}{l}\text { Garbage bin } \\
\text { (Ct value) }\end{array}$ & $\begin{array}{l}\text { Toilet seat } \\
\text { (Ct value) }\end{array}$ & $\begin{array}{c}\text { Patient's } \\
\text { mobile } \\
\text { phone }\end{array}$ & Bedside & Toliet \\
\hline 1 & 1 & $2021 / 8 / 4$ & $2021 / 8 / 20$ & 16 & Male & 70 & moderate & 0 & $(+) 26 / 28$ & $(-)$ & $(-)$ & $(-)$ & $(-)$ & $(+)-134$ & $(-)$ & $(-)$ & $(-)$ \\
\hline 2 & 1 & $2021 / 8 / 9$ & $2021 / 8 / 20$ & 11 & Female & 72 & moderate & 0 & $(+) 37 / 38$ & $(+) 24 / 32$ & $(-)$ & $(-)$ & $(-)$ & $(+)-134$ & $(-)$ & $(-)$ & $(-)$ \\
\hline 3 & 2 & $2021 / 8 / 11$ & $2021 / 8 / 20$ & 9 & Male & 29 & mild & 1 & $(+) 29 / 32$ & $(+) 33 / 33$ & $(+)-137$ & $(-)$ & $(-)$ & $(-)$ & $(-)$ & $(-)$ & $(-)$ \\
\hline 4 & 2 & $2021 / 8 / 12$ & $2021 / 8 / 20$ & 8 & Male & 37 & moderate & 2 & $(+) 33 / 36$ & $(+) 30 / 36$ & $(+)-139$ & $(-)$ & $(-)$ & $(-)$ & $(-)$ & $(-)$ & $(-)$ \\
\hline 5 & 3 & $2021 / 8 / 8$ & $2021 / 8 / 20$ & 12 & Male & 65 & moderate & 1 & $(+) 29 / 31$ & $(+) 30 / 39$ & $(+)-139$ & $(+) 38 /-$ & $(+) 28 / 29$ & (+) $35 /-$ & $(-)$ & $(-)$ & $(-)$ \\
\hline 6 & 3 & $2021 / 8 / 9$ & $2021 / 8 / 20$ & 11 & Female & 65 & moderate & 2 & $(+) 32 / 35$ & $(+) 35 / 35$ & $(-)$ & $(-)$ & $(+) 28 / 29$ & (+) $35 /-$ & $(-)$ & $(-)$ & $(-)$ \\
\hline 7 & 4 & $2021 / 8 / 9$ & $2021 / 8 / 20$ & 11 & Male & 46 & moderate & 0 & $(+) 27 / 28$ & $(-)$ & $(+)-136$ & $(-)$ & $(-)$ & $(-)$ & $(-)$ & $(-)$ & $(-)$ \\
\hline 8 & 5 & $2021 / 8 / 10$ & $2021 / 8 / 20$ & 10 & Male & 19 & moderate & 2 & $(+) 31 / 32$ & $(+) 35 / 36$ & $(-)$ & $(-)$ & $(-)$ & $(+)-140$ & $(-)$ & $(-)$ & $(-)$ \\
\hline 9 & 6 & $2021 / 8 / 11$ & $2021 / 8 / 20$ & 9 & Male & 49 & moderate & 2 & $(+) 30 / 32$ & $(+) 36 / 36$ & $(-)$ & $(-)$ & $(+) 33 / 29$ & (+) $36 /-$ & $(-)$ & $(-)$ & $(-)$ \\
\hline 10 & 7 & $2021 / 8 / 8$ & $2021 / 8 / 27$ & 19 & Female & 52 & moderate & 2 & $(+) 36 / 40$ & $(+)-140$ & (+) $35 /-$ & $(-)$ & $(-)$ & $(-)$ & $(-)$ & $(-)$ & $(-)$ \\
\hline
\end{tabular}

Figure 3 Information of 12 positive environmental specimens among 10 COVID-19 cases. (+) represents positive environmental surfaces specimens, and (-) represents negative environmental surfaces specimens. The number represents the cycle threshold (Ct) values.

Table 3 Factors associated with environmental contamination in COVID-19 cases

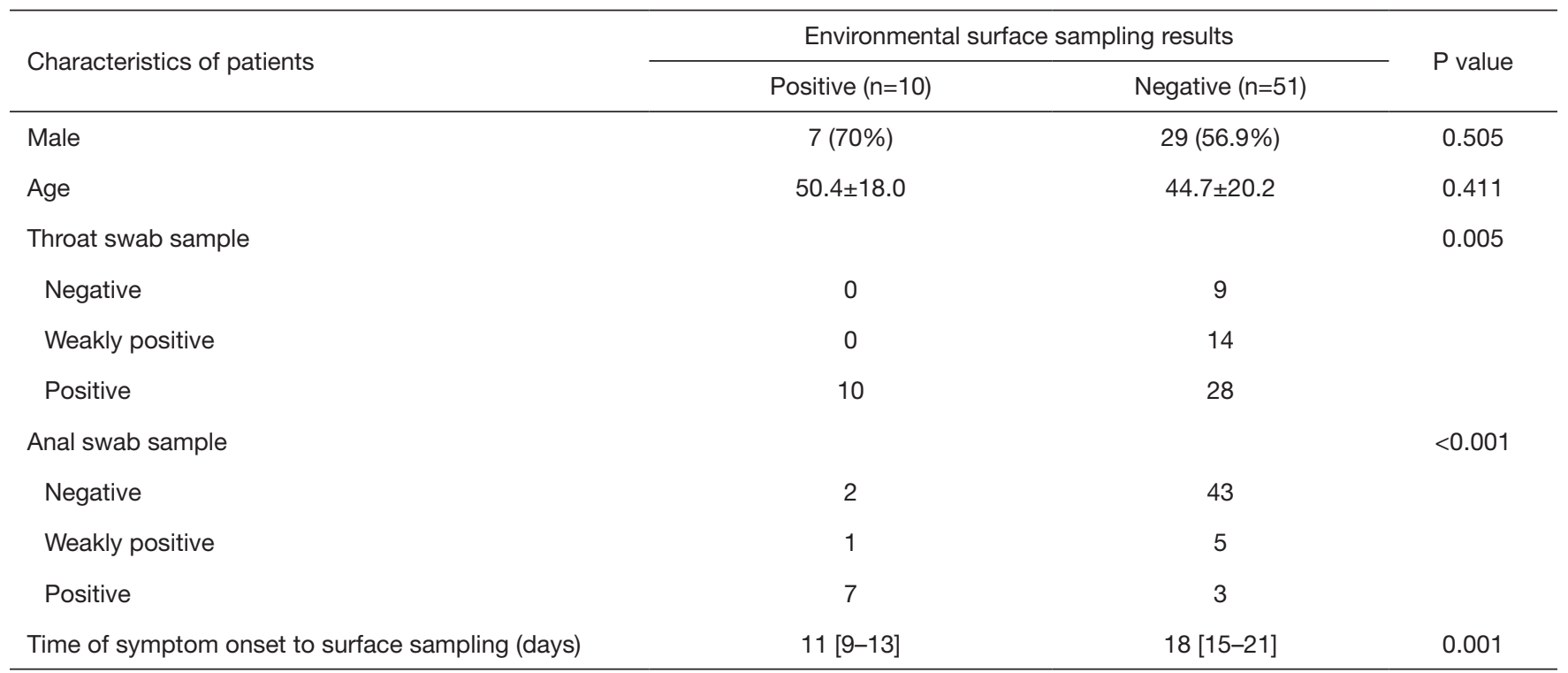

was also higher in the positive environment group than in the negative environment group $(70 \%$ vs. $5.9 \% ; \mathrm{P}<0.001)$. Interestingly, the time of symptom onset to surface sampling was shorter in the positive environment group than in the negative environment group (11 vs. 18 days; $\mathrm{P}=0.001$ ).

Multivariable GLMM was performed to identify risk factors associated with positive environmental sampling results (Table 4). The positive anal swab sample (OR 27.183; 95\% CI: 2.359-226.063; $\mathrm{P}=0.003)$ and the time from symptom onset to surface sampling (OR $0.801 ; 95 \% \mathrm{CI}$ : 0.501-0.990; $\mathrm{P}=0.046$ ) were found to be independently associated with the positive environmental sample.

\section{Characteristics of patient's hygiene behaviors}

Patients' hygiene behaviors are shown in Table 5. To be specific, $33.3 \%$ of the patients did not clean or disinfect their bedside tables or toilets, and $8.3 \%$ only cleaned their bedside tables or toilets. More than half of the cases never $(22.2 \%)$ or occasionally $(36.1 \%)$ disinfected their mobile phones every day. More than half of the cases often $(25 \%)$ or always $(30.6 \%)$ put masks on their bedside tables. Most of the cases $(63.9 \%)$ threw already-used masks into the garbage bin with a lid. However, $16.7 \%$ just threw alreadyused masks into a handy garbage bin, with or without a lid. 
Table 4 Multivariable generalized linear mixed model-identified risk factors associated with environmental contamination

\begin{tabular}{lcc}
\hline Item & OR & 95\% Cl \\
\hline Anal swab sample (negative) & 1 (referent) & - \\
Anal swab sample (weakly positive) & 2.017 & $0.267-37.265$ \\
Anal swab sample (positive) & 27.183 & 0.513 \\
Time from symptom onset to surface sampling & 0.801 & 0.003 \\
\hline
\end{tabular}

OR, odds ratio.

\section{Discussion}

In this study, we analyzed the samples from environmental surfaces and air contaminated by SARS-CoV-2 Delta VOC in hospital wards of COVID-19 cases. Toilet seats had the highest contamination rate, followed by bedside tables and garbage bins. The logistic regression analyses suggested that the positive anal swab and the time from symptom onset to surface sampling were independently associated with the positive environmental sample. The investigation of hygiene behaviors revealed lack of disinfection of toilets, bedside tables, and mobile phones, as well as improper disposal of used masks, to be possibly associated with environmental contamination.

The culprit of the COVID-19 outbreak in Nanjing in July 2021 was the Delta VOC. The Delta VOC has a stronger transmissibility, a higher viral load, and a quicker progression than does the wild-type strain (18). However, its effects on the environment are not clear. Our research showed that the Delta VOC was positive in samples mainly from toilets and bedside tables. The positive rates and surface distribution characteristics are in line with those previously reported $(19,20)$. The environmental contamination by asymptomatic cases with positive anal swab results was also mainly concentrated in toilets, and there could have been a chain of transmission with mobile phones as a medium (14). Interestingly, we found no positive cell phone specimens in our study, and our questionnaire showed that half of the patients did not have the habit of disinfecting cell phones. This may be related to the fact that the sampling was performed at more than 1 week after the onset. Another study (21) in Nanjing in 2020 reported that the environmental contamination was mainly in toilets and that the samples taken within 10 days after disease onset were positive.

In one study from Wuhan in 2020 (22), SARS-CoV-2 aerosol was detected at all 3 sampling sites in intensive care unit, with positivity rates of $35.7 \%(5 / 14)$ near air outlets,
$44.4 \%(8 / 18)$ in the wards, and $12.5 \%(1 / 8)$ in the doctor's offices. However, only air samples from the site near the general wards tested positive $(18.2 \%, 2 / 11)$. Surprisingly, another study (12) discovered that all the air samples from wards were negative, which is similar to our research results. Some experts (23) believe it is a mistake to think that negative SARS-CoV-2 in some air samples may prove the absence of airborne transmission because the airborne transmission of respiratory viruses may rely on many other factors (24).

Positive environmental areas, such as toilets, bedside tables, or garbage bins, are often frequently contacted. The toilet is often exposed to the virus via feces $(25,26)$, so the possibility of toilet contamination is high. The results of toilet surface sampling were highly consistent with those of anal swabs. Comparing our positive and negative environmental samples, the results of anal swab samples and the time from symptom onset to sampling showed significant statistical differences. A previous study (14) showed that for asymptomatic cases with positive anal swabs, the rate of positive environmental samples was much higher than that in patients with negative anal swabs, with the positive samples mainly being taken from toilets and bed rails. Our further regression analysis indicated that the positive anal swab and the time from symptom onset to sampling were independent risk factors for the positive environmental sample, suggesting that more attention should be paid to the results of anal swabs, especially at the early stage after symptom onset. Our findings demonstrated that the median time from symptom onset to positive environmental sampling was 11 days.

The results of our survey showed that $33.3 \%$ of patients did not have the habit of cleaning or disinfecting the bedside tables and toilets, and disinfection was mostly done by the medical staff, indicating that the patients' awareness of disinfection should be enhanced through efforts such as health education. Surprisingly, 2 positive 
Table 5 The characteristics of the patient's hygiene habits in hospital (N=36)

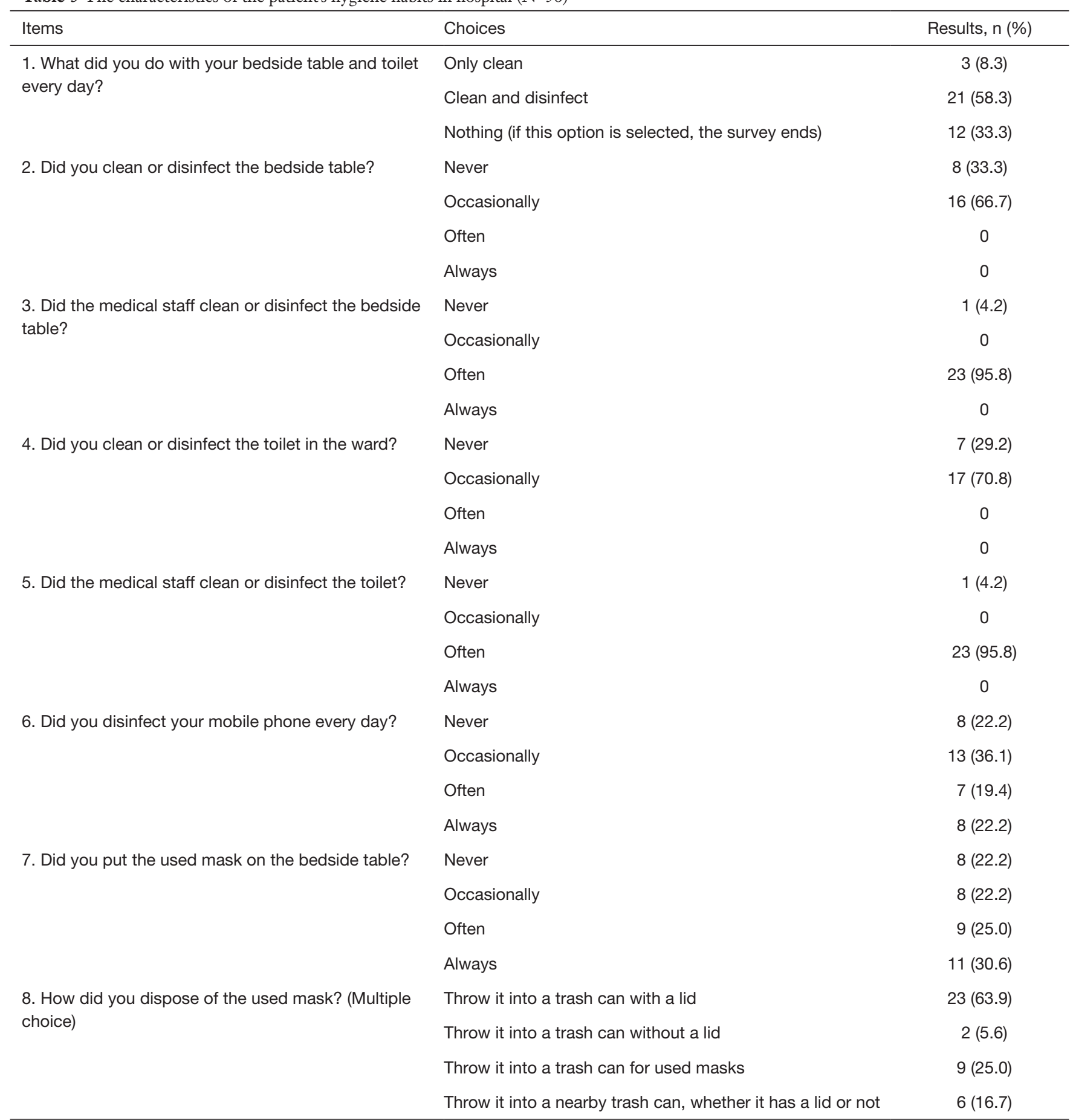

environmental samples, which had the lowest Ct values, were both collected from the garbage bins inside the ward. The possible reason is that more than half of the patients threw the used masks into the garbage bin inside the ward instead of the special garbage bin for used masks in the hallway. A previous study (27) reported that 13 of 31 (41.9\%) patients showed SARS-COV-2 positivity in both mask and nasopharyngeal swab samples (NPS), while 16 patients with 
negative results of the mask sampling had positive NPS results. Therefore, from the perspective of risk control, the garbage of the patients, such as used masks, needs careful management. Interestingly, up to $58.3 \%$ of patients did not disinfect their mobile phones every day. Although our experimental data included negative results in the surface samples from the mobile phone, the mobile phone is a possible transmission medium for the coronavirus (21). The negative results in our study may be attributed to the fact that the first environmental sampling was not conducted shortly after symptom onset.

Our study has some limitations. Although we collected 283 samples from 36 COVID-19 cases, the sample size of our study was relatively small. We only detected viral nucleic acid, and viral culture was not performed to test the viability. No environmental samples were collected from severe or serious COVID-19 patients, while environmental contamination was common in intensive care unit wards (22). Finally, our study lacks environmental sampling data collected shortly after onset (i.e., within 1 week after symptom onset). Despite these limitations, we still believe that our findings can be used to guide the prevention and control of COVID-19.

In conclusion, SARS-CoV-2 Delta VOC can be found on environmental surfaces, especially toilets and bedside tables, and can often be detected within a median time of 11 days from symptom onset. Our study provides potential predictors for environmental surface contamination, including positive anal swab and the time from symptom onset to sampling. The patients' awareness of disinfecting the environmental surfaces should be enhanced. Hopefully, our findings can guide improved infection control in the hospital.

\section{Acknowledgments}

We thank KEY Translation Studio (Nanjing, China) for the critical revising of the manuscript for English language and grammar.

Funding: None.

\section{Footnote}

Reporting Checklist: The authors have completed the Materials Design Analysis Reporting (MDAR) checklist. Available at https://dx.doi.org/10.21037/atm-21-5147

Data Sharing Statement: Available at https://dx.doi. org/10.21037/atm-21-5147
Conflicts of Interest: All authors have completed the ICMJE uniform disclosure form (available at https://dx.doi. org/10.21037/atm-21-5147). MC is scheduled to serve as an unpaid editorial board member of Annals of Translational Medicine from March 2021 to February 2022. The other authors have no conflicts of interest to declare.

Ethical Statement: The authors are accountable for all aspects of the work in ensuring that questions related to the accuracy or integrity of any part of the work are appropriately investigated and resolved. The study was conducted in accordance with the Declaration of Helsinki (as revised in 2013). Our study protocol was approved by the Ethics Committee of The No. 2 Hospital of Nanjing (No. 2021-ls-ky030). Informed consent was obtained from the patients before the questionnaire survey. Personal information was not obtained in this study.

Open Access Statement: This is an Open Access article distributed in accordance with the Creative Commons Attribution-NonCommercial-NoDerivs 4.0 International License (CC BY-NC-ND 4.0), which permits the noncommercial replication and distribution of the article with the strict proviso that no changes or edits are made and the original work is properly cited (including links to both the formal publication through the relevant DOI and the license). See: https://creativecommons.org/licenses/by-nc-nd/4.0/.

\section{References}

1. WHO. Weekly operational update on COVID-19. Available online: https://www.who.int/publications/ $\mathrm{m} /$ item/weekly-operational-update-on-covid-19---6september-2021

2. WHO. (2021a). Tracking SARS-CoV-2 variants. Available online: https://www.who.int/en/activities/tracking-SARSCoV-2-variants/

3. Mishra S, Mindermann S, Sharma M, et al. Changing composition of SARS-CoV-2 lineages and rise of Delta variant in England. EClinicalMedicine 2021;39:101064.

4. Li XN, Huang Y, Wang W, et al. Effectiveness of inactivated SARS-CoV-2 vaccines against the Delta variant infection in Guangzhou: a test-negative case-control realworld study. Emerg Microbes Infect 2021;10:1751-9.

5. Sheikh A, McMenamin J, Taylor B, et al. SARSCoV-2 Delta VOC in Scotland: demographics, risk of hospital admission, and vaccine effectiveness. Lancet 2021;397:2461-2. 
6. Hoffmann M, Hofmann-Winkler H, Krüger N, et al. SARS-CoV-2 variant B.1.617 is resistant to bamlanivimab and evades antibodies induced by infection and vaccination. Cell Rep 2021;36:109415.

7. Ong SWX, Chiew CJ, Ang LW, et al. Clinical and virological features of SARS-CoV-2 variants of concern: a retrospective cohort study comparing B.1.1.7 (Alpha), B.1.315 (Beta), and B.1.617.2 (Delta). Clin Infect Dis 2021. [Epub ahead of print]. doi: 10.1093/cid/ciab721.

8. Brown CM, Vostok J, Johnson H, et al. Outbreak of SARS-CoV-2 Infections, Including COVID-19 Vaccine Breakthrough Infections, Associated with Large Public Gatherings - Barnstable County, Massachusetts, July 2021. MMWR Morb Mortal Wkly Rep 2021;70:1059-62.

9. Hacisuleyman E, Hale C, Saito Y, et al. Vaccine Breakthrough Infections with SARS-CoV-2 Variants. N Engl J Med 2021;384:2212-8.

10. Chu DK, Akl EA, Duda S, et al. Physical distancing, face masks, and eye protection to prevent person-to-person transmission of SARS-CoV-2 and COVID-19: a systematic review and meta-analysis. Lancet 2020;395:1973-87.

11. Karia R, Gupta I, Khandait H, et al. COVID-19 and its Modes of Transmission. SN Compr Clin Med 2020. [Epub ahead of print]. doi: 10.1007/s42399-020-00498-4.

12. Wei L, Lin J, Duan X, et al. Asymptomatic COVID-19 Patients Can Contaminate Their Surroundings: an Environment Sampling Study. mSphere 2020;5:00442-20.

13. Ong SWX, Tan YK, Chia PY, et al. Air, Surface Environmental, and Personal Protective Equipment Contamination by Severe Acute Respiratory Syndrome Coronavirus 2 (SARS-CoV-2) From a Symptomatic Patient. JAMA 2020;323:1610-2.

14. Huang Y, Ding Z, Chen Q, et al. Environmental virus detection associated with asymptomatic SARS-CoV-2infected individuals with positive anal swabs. Sci Total Environ 2021;753:142289.

15. Cheng VC, Wong SC, Chan VW, et al. Air and environmental sampling for SARS-CoV-2 around hospitalized patients with coronavirus disease 2019 (COVID-19). Infect Control Hosp Epidemiol 2020;41:1258-65.

16. Zhou L, Yao M, Zhang X, et al. Breath-, air- and surface-borne SARS-CoV-2 in hospitals. J Aerosol Sci 2021;152:105693.

17. Tan M, Wang Y, Luo L, et al. How the public used face masks in China during the coronavirus disease pandemic:
A survey study. Int J Nurs Stud 2021;115:103853.

18. Wang Y, Chen R, Hu F, et al. Transmission, viral kinetics and clinical characteristics of the emergent SARS-CoV-2 Delta VOC in Guangzhou, China. EClinicalMedicine 2021;40:101129.

19. Luo L, Liu D, Zhang H, et al. Air and surface contamination in non-health care settings among 641 environmental specimens of 39 COVID-19 cases. PLoS Negl Trop Dis 2020;14:e0008570.

20. Liu J, Liu J, He Z, et al. Duration of SARS-CoV-2 positive in quarantine room environments: A perspective analysis. Int J Infect Dis 2021;105:68-74.

21. Ding Z, Qian H, Xu B, et al. Toilets dominate environmental detection of severe acute respiratory syndrome coronavirus 2 in a hospital. Sci Total Environ 2021;753:141710.

22. Guo ZD, Wang ZY, Zhang SF, et al. Aerosol and Surface Distribution of Severe Acute Respiratory Syndrome Coronavirus 2 in Hospital Wards, Wuhan, China, 2020. Emerg Infect Dis 2020;26:1583-91.

23. Greenhalgh T, Jimenez JL, Prather KA, et al. Ten scientific reasons in support of airborne transmission of SARS-CoV-2. Lancet 2021;397:1603-5.

24. Pan M, Lednicky JA, Wu CY. Collection, particle sizing and detection of airborne viruses. J Appl Microbiol 2019;127:1596-611.

25. Aboubakr HA, Sharafeldin TA, Goyal SM. Stability of SARS-CoV-2 and other coronaviruses in the environment and on common touch surfaces and the influence of climatic conditions: A review. Transbound Emerg Dis 2021;68:296-312.

26. Holshue ML, DeBolt C, Lindquist S, et al. First Case of 2019 Novel Coronavirus in the United States. N Engl J Med 2020;382:929-36.

27. Sriraman K, Shaikh A, Parikh S, et al. Non-invasive adapted N-95 mask sampling captures variation in viral particles expelled by COVID-19 patients: Implications in understanding SARS-CoV2 transmission. PLoS One 2021;16:e0249525.

Cite this article as: Chu M, Chu M, Mao Y, Li Z, Liu S, Liu H, Ge J, Peng Z, Yang Y, Liu Y, Tang J. Environmental sampling of the severe acute respiratory syndrome coronavirus 2 Delta variant in the inpatient wards of a hospital in Nanjing. Ann Transl Med 2021;9(23):1712. doi: 10.21037/atm-21-5147 